\title{
The effects of reduced rpd3 levels on fly physiology
}

\author{
Jared K. Woods ${ }^{\mathrm{a}}$, Tahereh Ziafazeli ${ }^{\mathrm{a}, \mathrm{c}}$ and Blanka Rogina ${ }^{\mathrm{a}, \mathrm{b}, *}$ \\ ${ }^{\mathrm{a}}$ Department of Genetics \& Genome Sciences, School of Medicine, University of Connecticut Health, \\ Farmington, CT, USA \\ ${ }^{\mathrm{b}}$ Institute for Systems Genomics, School of Medicine, University of Connecticut Health, Farmington, CT, USA \\ ${ }^{\mathrm{c}}$ Present address: Department of Pediatrics, Division of Pediatric Endocrinology, Faculty of Health \\ Sciences, McMaster University, ON, Canada
}

\begin{abstract}
.
BACKGROUND: Rpd3 is a conserved histone deacetylase that removes acetyl groups from lysine residues within histones and other proteins. Reduction or inhibition of Rpd3 extends longevity in yeast, worms, and flies. Previous studies in flies suggest an overlap with the mechanism of lifespan extension by dietary restriction. However, the mechanism of $r p d 3$ 's effects on longevity remains unclear.

OBJECTIVES: In this study we investigated how $r p d 3$ reduction affects fly spontaneous physical activity, fecundity, and stress resistance.

METHODS: We examined the effects of $r p d 3$ reduction on fly spontaneous physical activity by using population monitors, we determined female fecundity by counting daily egg laying, and we determined fly survivorship in response to starvation and paraquat.

RESULTS: In flies, $r p d 3$ reduction increases peak spontaneous physical activity of $r p d 3^{\text {def }}$ male flies at a young age but does not affect total 24 hour activity. Male and female $r p d 3^{\text {def }}$ mutants are more resistant to starvation on low and high calorie diets. In addition, increased resistance to paraquat was observed in females of one allele. A decrease in rpd3 levels does not affect female fecundity.

CONCLUSIONS: A decrease in rpd3 levels mirrors some but not all changes associated with calorie restriction, illustrated by an increased peak of spontaneous activity in $r p d 3^{d e f} /+$ heterozygous male flies but no effect on total spontaneous activity and fecundity.
\end{abstract}

Keywords: rpd3, dietary restriction, aging, Drosophila melanogaster

\section{Introduction}

Aging is characterized with progressive decline of physiological responses. Dietary restriction (DR) without malnutrition delays age-related pathophysiology and increases mean and maximal lifespan in a number of species [1,2]. DR affects many physiological processes, including mitochondrial function, preserved protein homeostasis, enhanced genomic

\footnotetext{
*Corresponding author: Blanka Rogina, Ph.D., Department of Genetics and Genome Sciences, Institute for Systems Genomics, School of Medicine, University of Connecticut Health, 263 Farmington Ave., Farmington, CT 06030-6403, USA. Tel.: +1 860679 8771; Fax: +1 860679 8345; E-mail: Rogina@uchc.edu.
}

stability, increased insulin sensitivity, and nutrient metabolism. DR improves organismal health, and it delays the development of Type 2 diabetes, cardiovascular disease, neurodegeneration, and cancer. Similar health benefits have been observed in humans on a strict DR regimen without malnutrition, as well as in short-term, randomized clinical trials [3]. The effects of DR depend on the timing and particular nutrients in diet [2]. Some of the possible mediators of beneficial effects of DR include the nutrient-sensing insulin/insulin-like signaling (IIS), the Tor signaling pathway, AMPK, HSF, and sirtuins [2].

Members of class I of the zinc-dependent histone deacetylase (HDAC) family are vital regulators 
of chromatin structure and gene expression [4-6]. Class I HDACs have a role in development, as well as in pathophysiology of human disorders. Mutations that affect the normal function of HDAC 1 have been implicated in development of human cancers, Alzheimer's, Huntington's, and Parkinson's diseases. Thus HDAC inhibitors have the potential to be used in therapy for cancer, neurodegenerative disorders, muscular atrophy, and heart disease [6-9]. Underexpression of Rpd3, the Drosophila HDAC1 homologue, is associated with a longer lifespan in yeast and fruit flies [10-15]. Males heterozygous for either the hypomorphic or null rpd3 mutations live $33 \%$ and $41 \%$ longer compared to controls, respectively [11]. Female flies heterozygous for the hypomorphic allele have a $52 \%$ longer lifespan, while females carrying the null mutation have a longer maximum but not median lifespan [11]. Longer lifespan was also observed in flies fed 4-phenylbutyrate (PBA), a histone deacetylase inhibitor [16]. Others and our lab have shown that longevity extension associated with reduced $r p d 3$ overlaps with longevity effects of a dietary restricted diet $[11,13-15,17$, 18]. This hypothesis was supported by findings that DR and rpd3 under-expression are not additive in their lifespan effects [11]. They also cause a similar change in transcription levels of $d S i r 2$, takeout, 4E-BP, and members of the insulin/insulin-like signaling (IIS) pathway such as InR, chico, and dFoxo $[11,13,15,17]$. It was also reported that levels of rpd3 mRNA are decreased and $d$ Sir 2 mRNA levels are increased in starved flies, while the opposite was found in flies when re-fed; they have increased levels of rpd3 and reduced $d$ Sir 2 mRNA levels [19]. Flies mutant for both $r p d 3$ and $d \operatorname{Sir} 2$ live shorter compared to rpd3 mutant flies, suggesting that dSir2 mediates some effects of rpd3's effects on fly physiology and longevity [13]. However, when longevity studies were done on diets with varying caloric content, rpd3 mutant flies live longer on some DR diet levels suggesting that $r p d 3$ and DR have distinct but interacting effects on fly longevity [14]. This suggests that the relationship between the mechanism of longevity observed in rpd3 flies and DR is more complex than previously thought.

\section{Materials and methods}

\subsection{Fly strains and maintenance}

rpd3-deficient $\left(r p d 3^{\operatorname{def24}} / T M 6, S b\right)$ and $r p d 3-$ hypomorphic $\left(r p d 3^{P-U T R} / T M 3, S b, S e r\right)$ flies and their genetic controls, were used in the experiments. Heterozygous $r p d 3^{P-U T R} /+$ and $r p d 3^{P-1.8} /+$ flies were $\mathrm{F} 1$ generation from the crosses between male $r p d 3^{P-U T R} / T M 3, S b, S e r$ or $r p d 3^{P-1.8} / T M 6, T b$ and $C S$ virgin females. Male $r p d 3^{d e f 24} / T M 6, S b$ flies were crossed to $y w$ females. Genetic controls for $r p d 3^{d e f}$ were progeny generated by crossing $\mathrm{F} 1 \mathrm{rpd} 3^{\mathrm{def}} / \mathrm{yw}$ littermates. $C S$ and $y w$ flies are wild type for the $r p d 3$ gene, thus flies heterozygous for $r p d 3^{\operatorname{def} 24} / \mathrm{yw}$, $r p d 3^{P-U T R} / \mathrm{CS}$ and $r p d 3^{P-1.8} / \mathrm{CS}$ are referred as $r p d 3^{d e f} /+, r p d 3^{P-U T R} /+$, and $r p d 3^{P-1.8} /+$ in the text. The hypomorphic $r p d 3^{P-U T R}$ allele has a P-element inserted in the 5'UTR region of the rpd3 gene, which affects expression throughout the fly's body. The control $r p d 3^{P-1.8}\left(r p d 3^{P-1.8} / T M 6, T b\right)$ allele has a P-element inserted $1.8 \mathrm{~Kb}$ upstream from the transcriptional start site, which only decreases expression in the eye [20]. Canton $S$ and $y w$ were kindly provided by the Bloomington Stock center. Flies were collected within 24 hours of eclosion and maintained using standard corn culture media, low calorie $(0.5 \mathrm{~N}$, $0.7 \mathrm{~N})$ or high calorie $(1.5 \mathrm{~N})$ diet in plastic vials. The caloric content of $0.5 \mathrm{~N}$ food is $50 \%$ that of the $1.0 \mathrm{~N}$ food. Flies were kept at $25^{\circ} \mathrm{C}$ in a humidified incubator with a 12 hour light-dark cycle. 25 males and 25 females are kept together in each vial, and they are passed to a fresh vial every Monday, Wednesday, and Friday. Fly diets were prepared as previously reported [21].

\subsection{Spontaneous physical activity}

Flies were aged until 10 or 40 days of age on a corn diet. 10 males or 10 females were placed in separate glass vials containing culture media. 3 vials per $r p d 3^{d e f} /+$ and their controls, and 4 vials per $r p d 3^{P-U T R} /+$ and $r p d 3^{P-1.8} /+$ control were used. These vials were placed in mobility monitors that counted every time a fly passed the top, middle, or bottom of the vial. Data were taken at the start of the following light cycle to assure the flies had acclimated to the new environment [21].

\subsection{Fecundity}

Male $r p d 3^{P-U T R} / T M 3, S b, S e r$ or $r p d 3^{P-1.8} / T M 6, T b$ were crossed to $C S$ virgin females. 1 female virgin of the same genotype $\left(C S, r p d 3^{P-U T R} / C S\right.$ or $\left.r p d 3^{P-1.8} / C S\right)$ was collected on $\mathrm{CO}_{2}$ and placed in individual plastic vials containing $0.5 \mathrm{~N}$ or $1.5 \mathrm{~N}$ agar medium $[22,23]$. The flies were passed to new vials 
daily and the number of eggs was counted. 10 vials were used for each genotypes and each diet.

\subsection{Starvation and paraquat resistance}

Flies were collected as described above and aged until 10 or 40 days of age. They were separated into vials of 20 males or 20 females and transferred into new vials containing 2 filter papers with $300 \mu$ of DI $\mathrm{H}_{2} \mathrm{O}$ for starvation studies. For paraquat resistance 20 flies were transfer into a vial containing filter paper soaked with $300 \mu \mathrm{L}$ of $20 \mathrm{mM}$ paraquat following initial starvation for 6 hours [24]. The number of dead flies was counted hourly during the day and twice overnight. Stress resistance data were analyzed by log-rank tests using the JMP 12 program. The total number of flies per experiment is listed in Tables 3 and 4.

\section{Results}

\subsection{The effect of rpd3 reduction on fy spontaneous locomotor activity}

To test if the mechanism of rpd3 reduction on fly longevity is similar to DR we investigated various aspects of Drosophila physiology in $r p d 3$ mutant flies and their genetic controls. We used two different heterozygous $r p d 3$ alleles due to embryonic lethality of homozygous $r p d 3$ mutant flies. We used rpd3 deficient, an $r p d 3$ null line, $\left(r p d 3^{\text {def }} /+\right)$ and their genetic control, F1 progeny of $r p d 3^{d e f} /+$ littermates. The levels of $r p d 3$ mRNA in $r p d 3^{d e f} /+$ is $50 \%$ lower compared to controls. We also used $r p d 3^{P-U T R} /+$ flies, an $r p d 3$ hypomorph, and $r p d 3^{P-1.8} /+$, which are their genetic controls and have $r p d 3$ reduction only in the eyes [20]. The presence of one copy of a P-element in $r p d 3^{P-U T R} /+$ flies results in $60 \%$ of $r p d 3$ mRNA levels compared to heterozygous $r p d 3^{P-1.8} /+$ control flies [11]. Locomotor activity reflects the functional activity of the nervous system. There is an age-related decline in the locomotor activity of flies [21,22]. One of the hallmarks of DR is increased spontaneous locomotor activity, and it is observed in multiple species $[21,22,25,26]$. This is thought to be due to an increased scavenging for food. In mice and flies Sirt1 and dSir2 mediate the increased locomotor activity associated DR, respectively [25-27]. To test if there is an overlap in the mechanism of DR and rpd3 mutation, we examined the spontaneous physical activity of rpd3 mutants and their controls. We used popu- lation monitors, which allow continuous monitoring of spontaneous physical activity of the population of 10 flies during longer time periods [21]. At 10 days of age, we found an increase in peak activity in $r p d 3^{d e f} /+$ male flies (Fig. 1A, Table 1). However, at an older age the peak activity in male $r p d 3^{d e f} /+$ flies was not different compared to controls (Fig. 1B, Table 1). Total 24 hour activity was not different at age 10 or 40 (Fig. 1C). Females $r p d 3^{d e f} /+$ flies showed little difference compared to their controls at 10 days of age (data not shown). A similar tendency toward increased peaks of spontaneous activity but no significant effect on the peak or on total 24 hour activity was observed in $r p d 3^{P-U T R} /+$ male and female flies when compared to controls at 10 days of age (Fig. 1D-F).

\subsection{The effect of reduced rpd3 on fly fecundity}

We have previously reported that $r p d 3$ reduction does not affect egg production in virgin $r p d 3^{P-U T R}$ $1+$ compared to $r p d 3^{P-1.8} /+$ female flies aged on a regular diet [11]. DR reduces fly fecundity due to re-allocation of the resources from reproduction to maintenance and repair $[22,28]$. Here we examined if feeding $r p d 3^{P-U T R} /+$ flies a high $(1.5 \mathrm{~N})$ or a low $(0.5 \mathrm{~N})$ calorie diet would affect their fecundity. We counted the number of eggs laid by flies heterozygous for wild type Canton $S(C S)$ and $r p d 3^{P-U T R} /+$ or $r p d 3^{P-1.8} /+$ (genetic control) daily during the first 10 days of life. $C S$ female flies were included for an additional comparison since they share $50 \%$ of the genetic background, and to illustrate the negative effect of $0.5 \mathrm{~N}$ diet on female fecundity. If $r p d 3$ reduction mimics DR completely, we would expect $r p d 3^{P-U T R} /+$ females to lay fewer eggs compared to controls on a $1.5 \mathrm{~N}$ diet, and even fewer on a $0.5 \mathrm{~N}$ diet. However, there was no difference in total egg number laid by $r p d 3^{P-U T R} / C S$ females compared to $r p d 3^{P-1.8}$ on $1.5 \mathrm{~N}$ diet, and only a minor tendency toward a lower number on $0.5 \mathrm{~N}$ diet $(p=0.053)$ (Fig. 2A, B, Table 2). Furthermore, no differences in fecundity between $r p d 3^{P-U T R} / C S$ and $C S$ were observed. Thus $r p d 3$ reduction does not affect female fecundity on either $0.5 \mathrm{~N}$ or $1.5 \mathrm{~N}$ diet.

\subsection{Reduction in rpd3 increases starvation resistance of male and female flies}

Increased starvation resistance is a phenotype found in many long-lived animals, yet its modification by DR remains a debatable topic. The first 

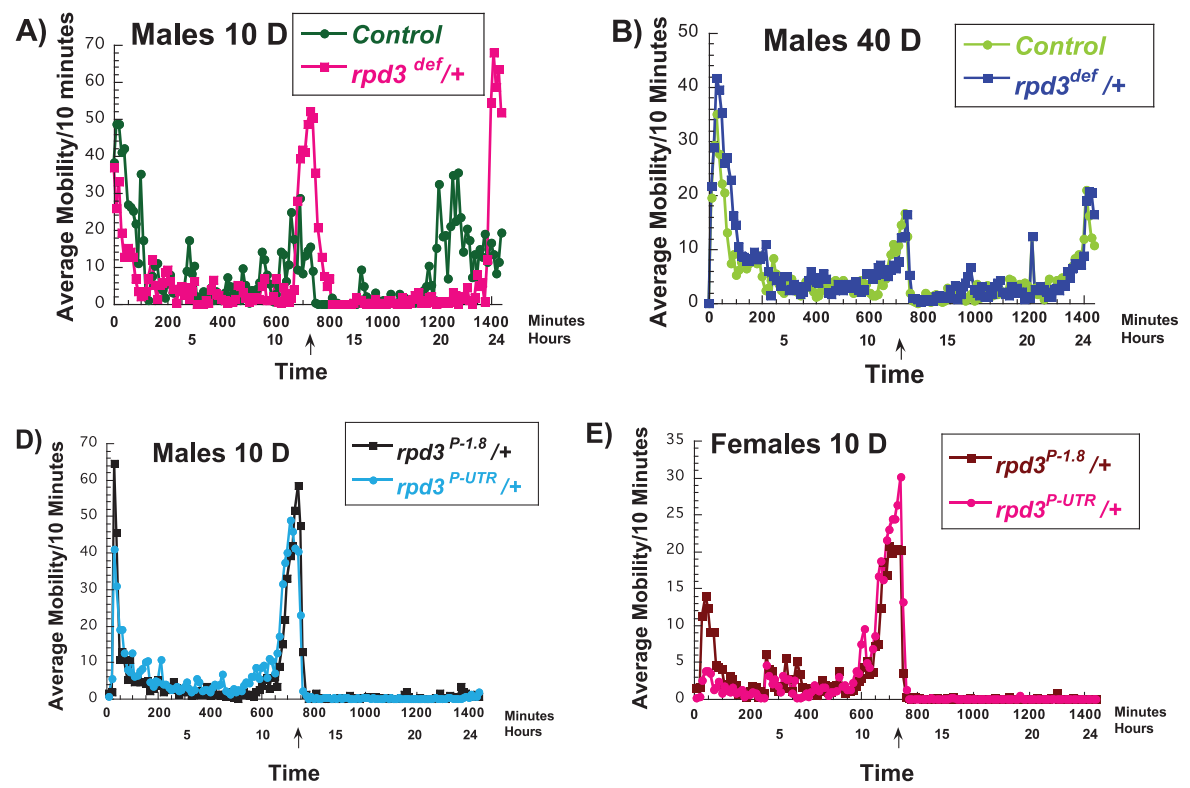

E)

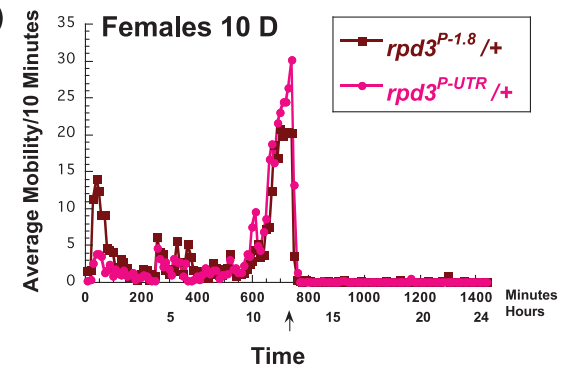

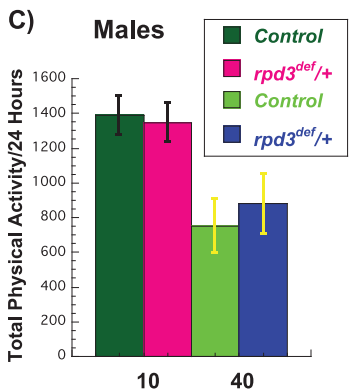

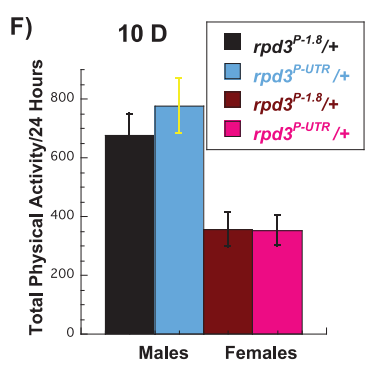

Fig. 1. Effects of rpd3 mutations on fly spontaneous physical activity. (A-C) Average (A, B) and total (C) 24 hours spontaneous physical

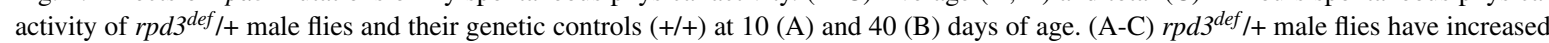
peak spontaneous physical activity compared to their genetic controls but the total locomotor activity of $r p d 3^{d e f} /+$ male flies over the course of 24 hours is simialr to their genetic controls at age 10 and 40 days. (D,E) Average spontaneous physical activity of $r p d 3^{P-U T R} /+$ male (D) and female (E) flies and their genetic controls $r p d 3^{P-1.8} /+$ at 10 days of age. (F) The total spontaneous physical activity of $r p d^{P-U T R} /+$ male and female flies and their genetic controls $r p d 3^{P-1.8} /+$ over the course of 24 hours at age 10 days. (A, B, D, E) The data are collected in 10 minutes bins and represent an average of sponteneous locomotor activity of three biological replicates of 10 female or 10 male flies (each replicate) of experimental ( $\left.r p d 3^{d e f} /+(\mathrm{A}-\mathrm{C}) ; r p d 3^{P-U T R}(\mathrm{D}-\mathrm{F})\right)$ or control (+/+(A-C); $r p d 3^{P-1.8}$ (D-F)) flies. An asterix on the $\mathrm{x}-\mathrm{axis}$ marks the time when the light was switched off and the transition to dark cycle (6:00PM or 720 minutes).

Table 1

Spontaneous physical activity of $r p d 3^{d e f}$ and $r p d 3^{P-U T R}$ heterozygous flies and controls

\begin{tabular}{|c|c|c|c|c|c|c|c|c|c|c|}
\hline \multirow[t]{2}{*}{ Gender } & \multirow[t]{2}{*}{ Genotype } & \multirow[t]{2}{*}{ Age } & \multicolumn{6}{|c|}{ Peak activity } & \multirow[t]{2}{*}{ Total activity } & \multirow[t]{2}{*}{$(\mathrm{SE})$} \\
\hline & & & 1 & $p$ & 2 & $p$ & 3 & $p$ & & \\
\hline M & $r p d 3^{d e f} /+$ & 10 & 141 & & 388 & & 192 & & 1347 & 112 \\
\hline M & Control & 10 & 223 & 0.3 & 171 & $0.004^{*}$ & 90 & 0.18 & 1390 & 305 \\
\hline M & $r p d 3^{d e f} /+$ & 40 & 193 & 85 & 93 & 879 & 172 & & & \\
\hline M & Control & 40 & 154 & 0.28 & 98 & 0.57 & 76 & 0.58 & 749 & 156 \\
\hline M & $r p d 3^{P-U T R} /+$ & 10 & 116 & 328 & 5 & 775 & 93 & & & \\
\hline M & $\operatorname{rpd} 3^{P-1.8} /+$ & 10 & 137 & 0.6 & 331 & 0.95 & 6 & 0.08 & 677 & 79 \\
\hline $\mathrm{F}$ & $r p d 3^{P-U T R} /+$ & 10 & 14 & 199 & 0 & 351 & & 51 & & \\
\hline $\mathrm{F}$ & $\operatorname{rpd} 3^{P-1.8} /+$ & 10 & 49 & 0.08 & 153 & 0.5 & 0 & 1.0 & 355 & 59 \\
\hline
\end{tabular}

The peaks and the total spontaneous physical activity of $r p d 3^{d e f} /+$ and their genetic controls $+/+, r p d^{P-U T R} /+$ and their genetic controls $r p d 3^{P-1.8} /+$. The peak 1 is sum of the total activity between 6AM-7AM, peak 2 between 5PM-7PM, and 3 between 5AM-6AM. The total spontenaous activity is sum of the activity over the course of 24 hours at age 10 or 40 days. The data were collected for at least three biological replicates of 10 female or 10 male flies (each replicate) of experimental or control flies. Student $T$ test analysis was used to determine the difference in the peaks of activity. $*=$ a statistical significant result.

Drosophila DR experiment found that both genders of flies had increased starvation resistance on lowcalorie food, yet the increase was not as great in males [28]. Reducing the calorie content in food, especially protein, increases starvation resistance in flies due to lipid accumulation [28, 29, 30]. Nevertheless, extended DR reduces starvation resistance in flies [28]. In addition, some studies indicate that genetic manipulation that mimics DR decreases starvation resistance because of the reduction in energy 


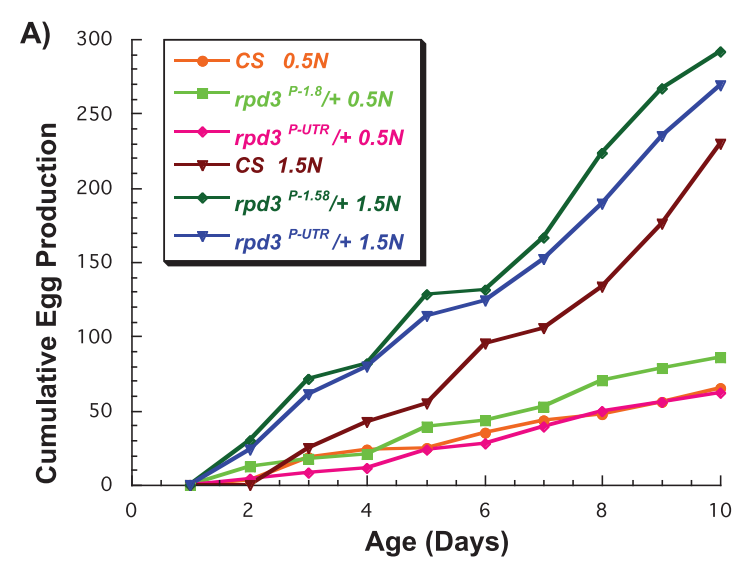

B)

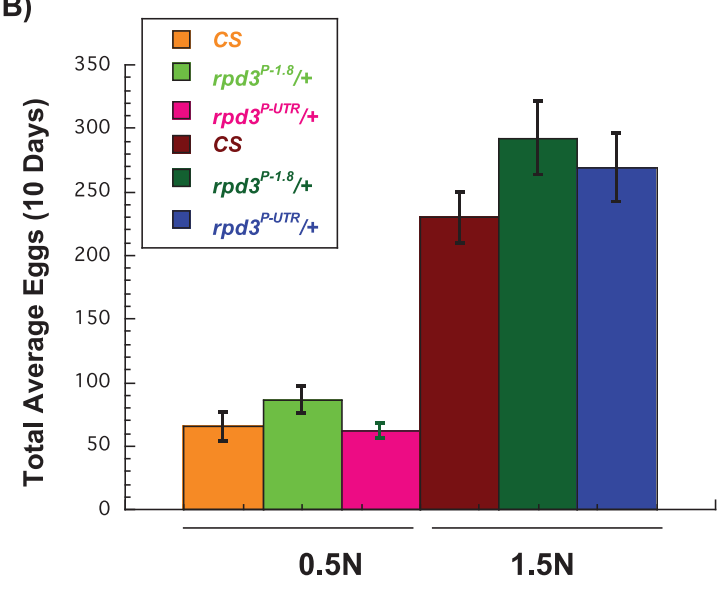

Fig. 2. Effect of rpd3 reduction on fly fecundity. (A) The average number of eggs per day laid by wild type Canton-S $(C S)$, $r p d 3^{P-U T R} / C S$, and genetic controls $r p d 3^{P-1.8} / \mathrm{CS}$ on $0.5 \mathrm{~N}$ and $1.5 \mathrm{~N}$ diets. (B) The total number of eggs per 10 day period laid by $C S, r p d 3^{P-U T R} / C S$, and $r p d 3^{P-1.8} / \mathrm{CS}$ on $0.5 \mathrm{~N}$ and $1.5 \mathrm{~N}$ diets. $r p d 3^{P-U T R} / C S$ flies laid similar numbers of eggs on $1.5 \mathrm{~N}$ but lower numbers on $0.5 \mathrm{~N}$ compared to the controls, a difference that did not reach significance $(p=0.053)$. 10 vials with a single male and a single female were used per genotype and diet.

Table 2

Effect of $r p d 3$ reduction on female fecundity

\begin{tabular}{lccr}
\hline Genotype & Food & Total eggs & SE \\
\hline$r p d 3^{P-U T R} /+$ & $0.5 \mathrm{~N}$ & 62 & 6.2 \\
$r p d 3^{P-1.8} /+$ & $0.5 \mathrm{~N}$ & 86 & 10.9 \\
$\mathrm{CS}$ & $0.5 \mathrm{~N}$ & 65 & 11.6 \\
$r p d 3^{P-U T R} /+$ & $1.5 \mathrm{~N}$ & 269 & 26.1 \\
$r p d 3^{P-1.8} /+$ & $1.5 \mathrm{~N}$ & 292 & 28.7 \\
$\mathrm{CS}$ & $1.5 \mathrm{~N}$ & 230 & 20.0 \\
\hline
\end{tabular}

The total number of eggs per 10 day period laid by $C S$, $r p d 3^{P-U T R} / C S$, and $r p d 3^{P-1.8} / C S$ flies aged on $0.5 \mathrm{~N}$ or $1.5 \mathrm{~N}$ diets. 10 vials with a single male and a single female were used per genotype and diet. stores [31]. $r p d 3^{d e f} /+$ and control $(+/+)$ flies were raised on different food levels until 10 or 40 days of age and then exposed to starvation. We used $0.7 \mathrm{~N}$ as a DR diet and $1.5 \mathrm{~N}$ as a high calorie diet. We first compared the starvation resistance of aging control flies on $0.7 \mathrm{~N}$ to controls aged on $1.5 \mathrm{~N}$ food. At 10 days of age male and female control flies aged on $1.5 \mathrm{~N}$ food had a higher starvation resistance compared to flies kept on $0.7 \mathrm{~N}$ food (Fig. 3A,B, Table 3). However, at 40 days of age, there were no differences in starvation resistance for flies aged on $0.7 \mathrm{~N}$ or $1.5 \mathrm{~N}$ food (Fig. 3C,D, Table 3). Another interesting observation was that control male flies aged on $0.7 \mathrm{~N}$ or $1.5 \mathrm{~N}$ had a much lower starvation resistance compared to control females aged on the same food at 10 days of age. However, both male and female control flies had an almost identical starvation resistance at 40 days of age regardless of the content of the food on which they were aged (Fig. 3E). Male and female $r p d 3^{d e f} /+$ flies were more resistant to starvation than $+/+$ males at $0.7 \mathrm{~N}$ and $1.5 \mathrm{~N}$ (Fig. 3A-D, Table 3). In addition, starvation resistance in female $r p d 3^{d e f} /+$ was much higher compared to males at both 10 and 40 days of age. Thus $r p d 3$ reduction increases starvation resistance in $r p d 3^{d e f} /+$ flies when aged on a standard, a low, or a high calorie diet.

\subsection{The effect of reduced rpd3 mRNA levels on paraquat resistance}

Increased resistance to oxidative stress is often associated with increased longevity and it has been suggested that improved stress response could be one of the contributors to longer life [24]. DR does not affect oxidative stress resistance of young flies but has a negative effect on aged flies [28]. We have reported that $r p d 3$ reduction increases resistance to hydrogen peroxide, but this resistance depends on fly age and sex. Resistance is higher in females compared to males, and more pronounced at older ages [18]. Increased resistance to paraquat is also observed in flies with heart-specific rpd3 reduction, as well as whole body reduction in 2 days old flies [15]. However, the effect of whole body rpd3 reduction on paraquat resistance during aging has not been determined. There is an age-associated decline in resistance to paraquat in wild type flies, thus we examined if rpd3 reduction could postpone the known age-associated decline in resistance [32]. The response of $r p d 3^{d e f} /+$ and $r p d 3^{P-U T R} /+$ flies to paraquat depends on fly sex, age, and allele. 

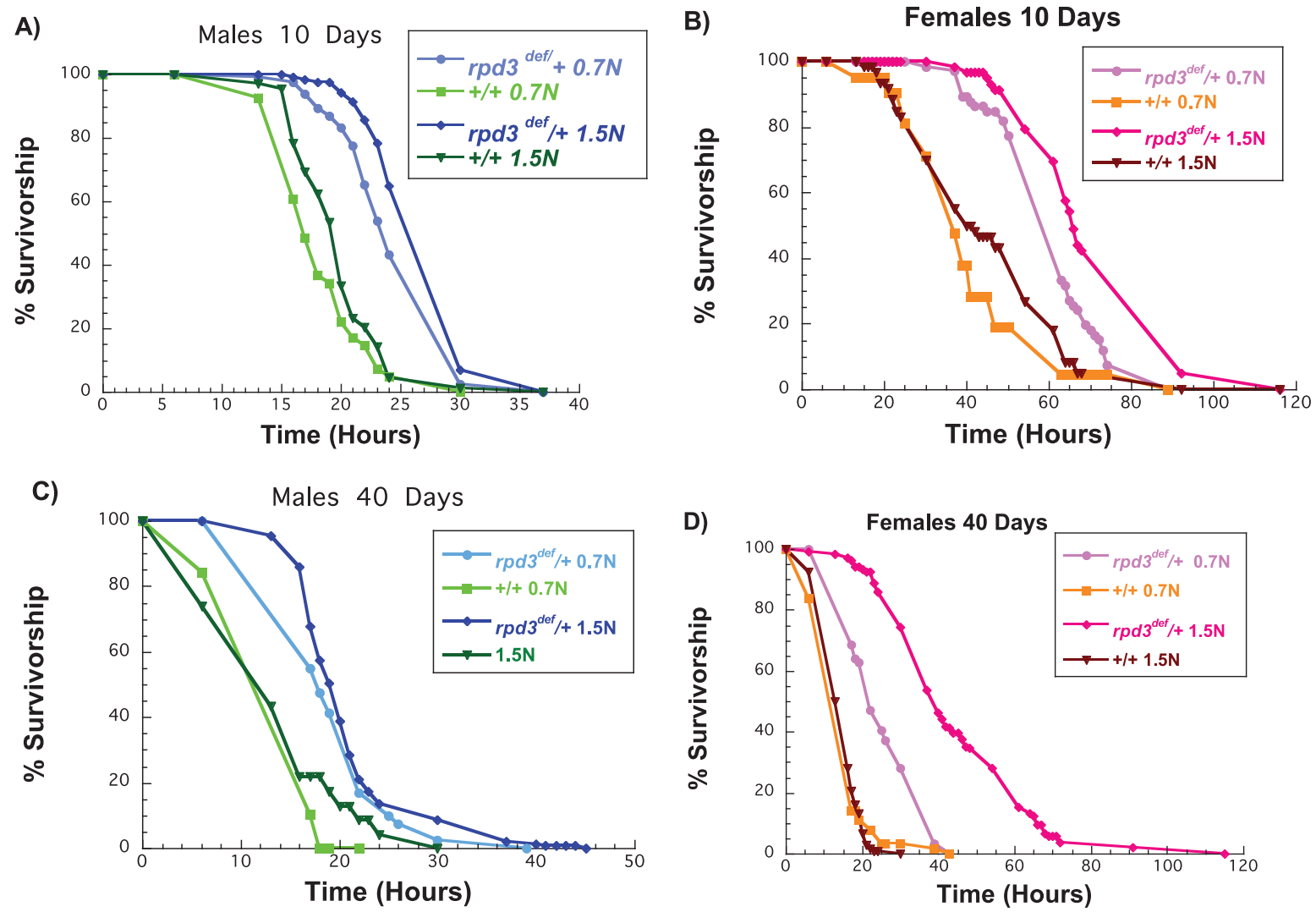

Fig. 3. Reduction of $r p d 3$ increases resistance to starvation of male and female flies on a low and a high calorie diet. (A, B) Survival curves of $r p d 3^{\text {def }} /+$ and $+/+$ male (A) and female (B) flies aged on $0.7 \mathrm{~N}$ or $1.5 \mathrm{~N}$ food levels and exposed to starvation at 10 days of age. (C, D) Survival curves of $r p d 3^{d e f} /+$ and+/+male (C) and female (D) flies aged on $0.7 \mathrm{~N}$ or $1.5 \mathrm{~N}$ food levels and exposed to starvation at 40 days of age.

$r p d 3^{P-U T R} /+$ females had increased resistance to paraquat at both 10 and 40 days, while males had no difference compared to controls (Fig. 4A, B). In contrast, $r p d 3^{d e f} /+$ females were less resistant to paraquat at 10 days while there was no difference at age 40 . There was no difference in resistance to paraquat in $r p d 3^{d e f} /+$ males at both ages (Fig. 4C, D). These data illustrate how genetic background, level of $r p d 3$ reduction, age, and sex affect fly resistance to paraquat.

\section{Discussion}

DR has multiple effects on physiology. In flies, DR reduces fecundity, increases fly spontaneous physical activity, increases mitochondrial biogenesis, and slows down age-related mortality [22, 28, 33]. The effects of $r p d 3$ reduction on lifespan in flies appear to at least partially overlap with the effects of DR, as DR and $\operatorname{rpd} 3$ whole body under-expression share a key effect on dSir2, takeout, chico, InR, and dFoxo levels of transcription [11, 13-15, 17-19]. Heart-specific rpd3 reduction increases the levels of Sod2, dSir2, dFoxo, Thor, and DptB in 2 day old flies [15]. dSir2 has been thought to mediate longevity extension in DR flies, illustrated by a lack of DR longevity effects in $d$ Sir 2 mutant flies. Flies double mutant for $d \operatorname{Sir} 2$ and $r p d 3$ live shorter compared to single rpd3 mutant flies suggesting that dSir2 mediates at least partially the longevity effect in rpd3 flies [13]. In addition, $r p d 3$ reduction and DR are not additive in their effects on lifespan [11]. Therefore, it was hypothesized that the mechanism of longevity extension in DR and rpd3 flies is the same or related [11, 13]. However, rpd3 mutants live longer under conditions close to starvation and during starvation, suggesting that DR and $r p d 3$ may have distinct but interacting or overlapping effects on fly longevity $[14,18]$. To gain further insight into the mechanism of lifespan extension observed in rpd3 flies, we determined if similar 
Table 3

Starvation resistance of $r p d 3^{d e f} /+$ heterozygous flies and genetic controls

\begin{tabular}{|c|c|c|c|c|c|c|c|}
\hline Gender & Genotype & $\mathrm{N}$ & Age & Food & Mean LS (\% change) & $X^{2}$ & $p$ \\
\hline M & $r p d 3^{d e f} /+$ & 115 & 10 & $0.7 \mathrm{~N}$ & 25 & 66.054 & $<0.0001^{*}$ \\
\hline M & Control & 41 & 10 & $0.7 \mathrm{~N}$ & $19(-31.5)$ & & \\
\hline $\mathrm{F}$ & $r p d 3^{d e f} /+$ & 104 & 10 & $0.7 \mathrm{~N}$ & 45 & 15.075 & $<0.0001^{*}$ \\
\hline $\mathrm{F}$ & Control & 8 & 10 & $0.7 \mathrm{~N}$ & $38(-18.4)$ & & \\
\hline M & $\operatorname{rpd} 3^{\text {def } /+}$ & 126 & 10 & $1.5 \mathrm{~N}$ & 28 & 124.6 & $<0.0001^{*}$ \\
\hline M & Control & 69 & 10 & $1.5 \mathrm{~N}$ & $20(-40)$ & & \\
\hline $\mathrm{F}$ & $r p d 3^{d e f} /+$ & 117 & 10 & $1.5 \mathrm{~N}$ & 75 & 95.77 & $<0.0001^{*}$ \\
\hline $\mathrm{F}$ & Control & 100 & 10 & $1.5 \mathrm{~N}$ & $46(-63)$ & & \\
\hline M & $r p d 3^{d e f} /+$ & 82 & 40 & $0.7 \mathrm{~N}$ & 20 & 23.267 & $<0.0001^{*}$ \\
\hline M & Control & 19 & 40 & $0.7 \mathrm{~N}$ & $15(-33)$ & & \\
\hline $\mathrm{F}$ & $r p d 3^{\text {def } /+}$ & 89 & 40 & $0.7 \mathrm{~N}$ & 26 & 44.512 & $<0.0001^{*}$ \\
\hline $\mathrm{F}$ & Control & 63 & 40 & $0.7 \mathrm{~N}$ & $17(-52)$ & & \\
\hline M & $r p d 3^{\text {def } /+}$ & 137 & 40 & $1.5 \mathrm{~N}$ & 21 & 23.01 & $<0.0001^{*}$ \\
\hline M & Control & 23 & 40 & $1.5 \mathrm{~N}$ & $14(-50)$ & & \\
\hline $\mathrm{F}$ & $r p d 3^{d e f} /+$ & 136 & 40 & $1.5 \mathrm{~N}$ & 45 & 261.77 & $<0.0001^{*}$ \\
\hline $\mathrm{F}$ & Control & 106 & 40 & $1.5 \mathrm{~N}$ & $15(-200)$ & & \\
\hline
\end{tabular}

The mean lifespan of $r p d 3^{\text {def } /+}$ and genetic control $(+/+)$ heterozygous male $(\mathrm{M})$ and female $(\mathrm{F})$ flies after exposure to starvation at 10 or 40 days of age. The flies were aged on $0.7 \mathrm{~N}$ or $1.5 \mathrm{~N}$ food levels before being exposed to starvation as indicated. Control values are compared to either male or female $r p d 3^{d e f} /+$ groups to determine the percent change in mean lifespan. Mean lifespans are in hours. Log-rank analyses were performed using the JMP 12 program. $\mathrm{M}=$ Males, $\mathrm{F}=$ Females, $\mathrm{N}=$ number of flies in the experiment. $*=$ a statistical significant result.

Table 4

$r p d 3^{P-U T R}$ but not $\operatorname{rpd} 3^{d e f}$ heterozygous female flies have higher paraquat resistance compared to the control flies in the same genetic backgrounds

\begin{tabular}{lccccccc}
\hline Gender & Genotype & $\mathrm{N}$ & Age & $\begin{array}{c}\text { Mean } \\
\text { (\% change })\end{array}$ & $X^{2}$ & $p$ & $\begin{array}{c}\text { Maximal lifespan } \\
\text { (\% change) }\end{array}$ \\
\hline $\mathrm{M}$ & $r p d 3^{\text {def } /+}$ & 71 & 10 & 24 & 8.7859 & $<0.0030^{*}$ & 43 \\
$\mathrm{M}$ & Control & 51 & 10 & $20(-20)$ & & & $25(-72)$ \\
$\mathrm{F}$ & $r p d 3^{\text {def } /+}$ & 70 & 10 & 31 & 9.0113 & $<0.0027^{*}$ & 67 \\
$\mathrm{~F}$ & Controls & 94 & 10 & $39(21)$ & & & $70(4)$ \\
$\mathrm{M}$ & $r p d 3^{\text {def } /+}$ & 49 & 40 & 11 & 0.5829 & 0.4633 & 17 \\
$\mathrm{M}$ & Control & 17 & 40 & $12(0.8)$ & & & $17(0)$ \\
$\mathrm{F}$ & $r p d 3^{\text {def } /+}$ & 28 & 40 & 16 & 0.0192 & 0.8898 & 24 \\
$\mathrm{~F}$ & Controls & 55 & 40 & $17(0.5)$ & & & $25(0)$ \\
$\mathrm{M}$ & $r p d 3^{P-U T R} /+$ & 172 & 10 & 32 & 7.0464 & $0.0079^{*}$ & 54 \\
$\mathrm{M}$ & $r p d 3^{P-1.8 /+}$ & 107 & 10 & $36(11)$ & & & $66(18)$ \\
$\mathrm{F}$ & $r p d 3^{P-U T R} /+$ & 172 & 10 & 52 & 29.548 & $<0.0001^{*}$ & 84 \\
$\mathrm{~F}$ & $r p d 3^{P-1.8 /+}$ & 121 & 10 & $42(-24)$ & & & $66(-27)$ \\
$\mathrm{M}$ & $r p d 3^{P-U T R} /+$ & 300 & 40 & 16 & 7.757 & $<0.0053^{*}$ & 18 \\
$\mathrm{M}$ & $r p d 3^{P-1.8} /+$ & 306 & 40 & $17(0.5)$ & & & $19(0.5)$ \\
$\mathrm{F}$ & $r p d 3^{P-U T R} /+$ & 160 & 40 & 24 & 94.416 & $<0.001^{*}$ & 42 \\
$\mathrm{~F}$ & $r p d 3^{P-1.8 /+}$ & 169 & 40 & $17(-41)$ & & & $24(-75)$ \\
\hline
\end{tabular}

The mean and maximal lifespan of $r p d 3^{d e f} /+$ and genetic control $(+/+)$, and $r p d 3^{P-U T R} /+$ and their genetic control $\left(r p d 3^{P-1.8} /+\right)$ heterozygous male $(\mathrm{M})$ and female $(\mathrm{F})$ flies after exposure to paraquat at 10 or 40 days of age. Control values are compared to either male or female $r p d 3^{d e f} /+$ groups or $r p d 3^{P-U T R}$ to determine the percent change in mean and maximal lifespan. Mean and maximal lifespan are in days. $\mathrm{N}=$ number of flies in the experiment. Log-rank analyses were performed using the JMP 12 program. * = a statistical significant result.

changes in physiology associated with DR can be observed in flies with reduced rpd3 levels. Particularly, we investigated the effects of decreased levels of $r p d 3$ on spontaneous physical activity, fecundity, and stress resistance. $r p d 3^{d e f} /+$ male flies had an increased peak in spontaneous activity at a younger age most likely mediated by increased dSir2 levels. Fly spontaneous physical activity decreases with 
A)

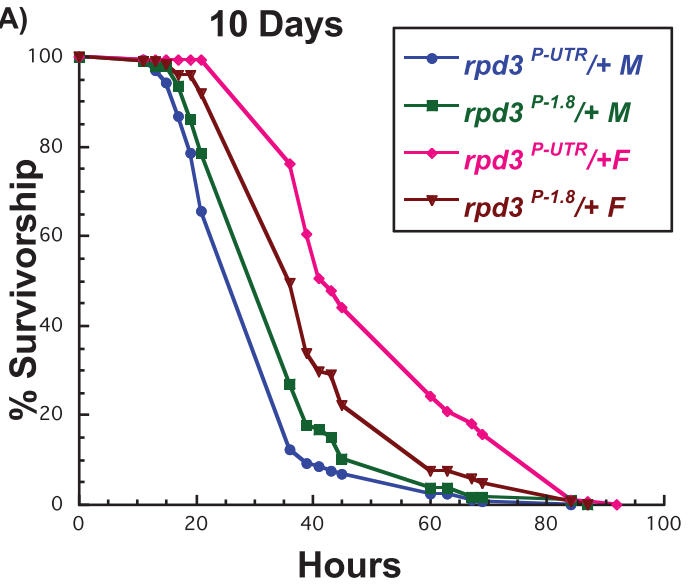

C)

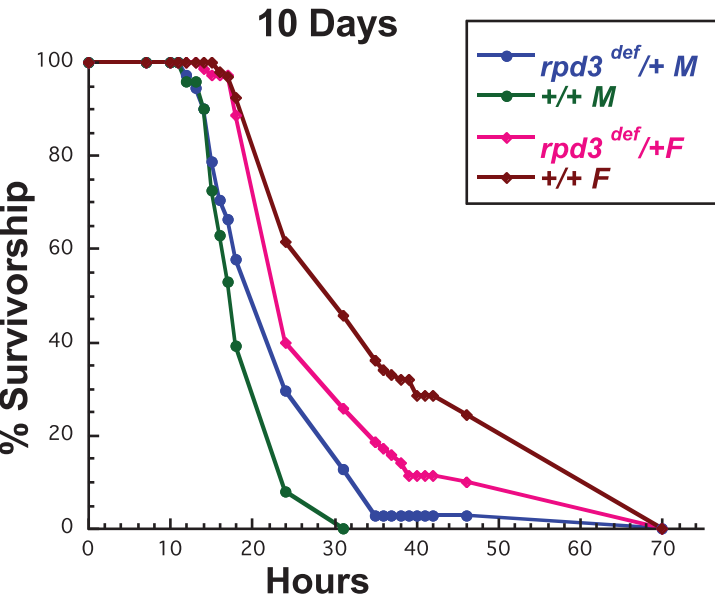

B)

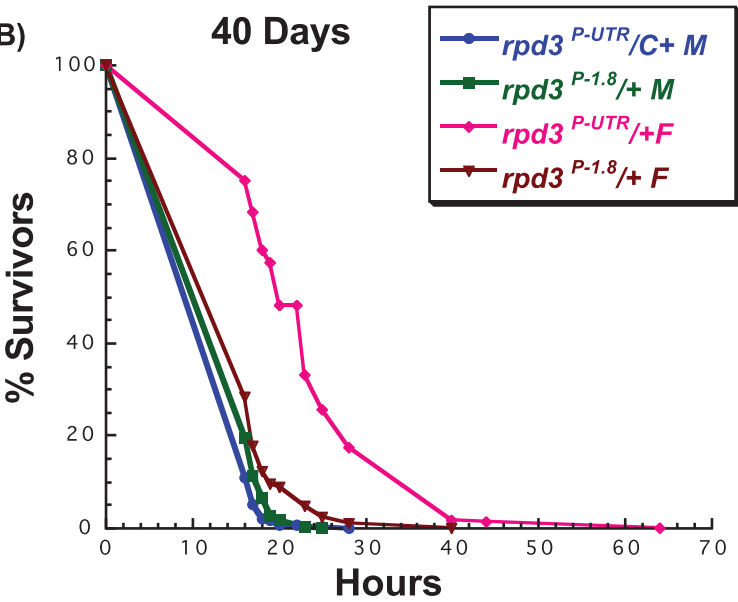

D)

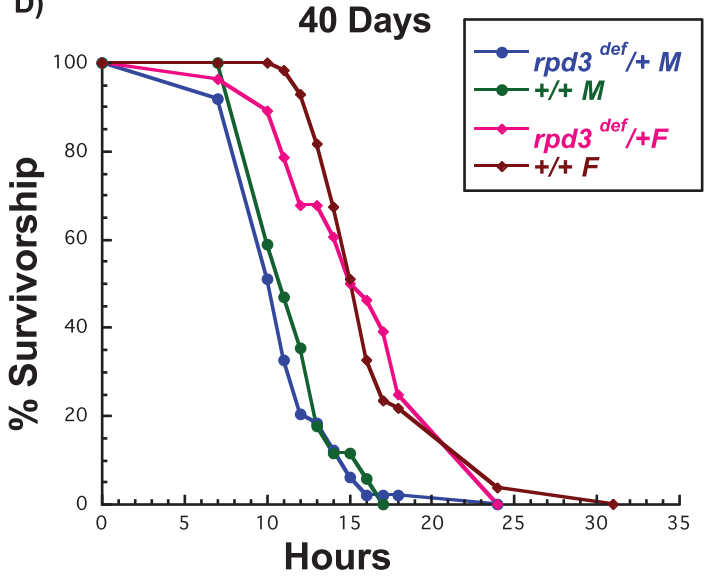

Fig. 4. $r p d 3$ reduction affects stress resistance in flies. (A, B) Survival curves for male and female $r p d 3^{P-U T R} /+\left(\right.$ experimental) and $r p d 3^{P-1.8} /+$ (control) flies exposed to $20 \mathrm{mM}$ paraquat at age 10 (A) or 40 (B) days. $r p d 3^{P-U T R} /+$ females are more resistant to paraquat at 10 and 40 days, but no changes in resistance were observed in male $r p d 3^{P \text {-UTR }} /+$ flies. (C, D) Survival curves for male and female $r p d 3^{d e f} /+$ and control flies

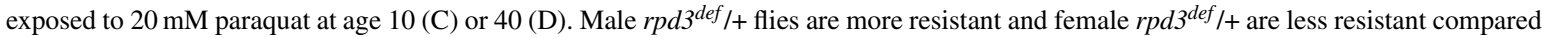
to control flies at age 10 . No difference in paraquat resistance was observed between $r p d 3^{d e f} /+$ male and female and control flies at 40 days.

age, and so do the differences in the peak activity. No difference was observed in $r p d 3^{d e f} /+$ males at age 40. Although peak activity appears to be associated with $r p d 3$ reduction in male $r p d 3^{d e f} /+$ flies at a younger age, no clear difference in peak or total spontaneous physical activity was associated with rpd3 reduction in $r p d 3^{d e f} /+$ females or $r p d 3^{P-U T R} /+$ male and female flies. The two rpd3 alleles have different levels of rpd3 mRNA that could explain difference in the peak of spontaneous physical activity or resistance to paraquat. While $r p d 3 \mathrm{mRNA}$ expression is reduced to $50 \%$ in the $r p d 3^{d e f} /+$ heterozygous flies compared to controls, the level of $r p d 3$ mRNA is reduced by $40 \%$ in $r p d 3^{P-U T R} /+$ compared to $r p d 3^{P-1.8} /+$ flies. The levels of $r p d 3$ mRNA are linked to different physiological consequences, as well as longevity effects. For instance, while males of both $r p d 3$ alleles and female $r p d 3^{P-U T R} /+$ flies have dramatically extended longevity, $r p d 3^{d e f} /+$ females only have extended maximal lifespan but not median lifespan [11]. Similarly, different effects on starvation resistance were observed in flies with whole body rpd3 reduction using RNAi mediated silencing compared to reduction only in fly heart tissue [15]. Likewise, the two rpd3 alleles respond differently to paraquat. However, we show that $\mathrm{H}_{2} \mathrm{O}_{2}$ and starvation resistance were similar in both $r p d 3$ alleles (discussed below).

DR has a negative effect on egg production in female flies due to a shift in use of available 
resources from reproduction to maintenance [25, 28]. If $r p d 3$ reduction mimics DR we would expect lower fecundity in rpd3 females aged on a DR diet. However, no difference in fecundity was associated with $r p d 3$ reduction. No difference in fecundity could be explained by higher energy storage and/or increased adaptation to DR found in rpd3 flies, as discussed below [18].

The effects on starvation and oxidative resistance caused by DR of wild type flies changes with age [28]. While DR increases starvation resistance early in life, the opposite was found in aged DR flies [28]. Increased resistance to starvation of young flies is associated with increased lipid content. However, this increased lipid storage is not sustained over longer time, which results in lower starvation resistance in older flies [28]. We previously reported that $r p d 3^{d e f} /+$ and $r p d 3^{P-U T R} /+$ flies have increased starvation resistance [18]. Heart-specific rpd3 reduction also increases starvation resistance in flies at 2 days of age [15]. Nevertheless, the same authors did not find increased starvation resistance in flies when $r p d 3$ was reduced in other tissues. Here we examined how aging rpd3 or control flies on $0.5 \mathrm{~N}$ or $1.5 \mathrm{~N}$ affect their starvation resistance. Our control flies aged on $1.5 \mathrm{~N}$ food had a small increase in starvation resistance compared to flies aged on $0.5 \mathrm{~N}$ food at an early age. However, no difference in starvation resistance between control male or female flies was observed at age 40 days, and diet did not have an effect at this age either. We observed increased starvation resistance in both $r p d 3$ alleles, with females showing a larger resistance compared to male $r p d 3$ flies. An increase in lipid storage caused by an increase in the activity of the enzymes associated with lipid biogenesis is often associated with increased starvation resistance [28]. Increased metabolic reserves in the form of both lipids and carbohydrates are associated with increased starvation resistance in an outbred population of Drosophila melanogaster [34]. We recently reported that $r p d 3$ reduction is associated with increased levels of triglycerides before and after starvation, which is at least partially mediated by reduced IIS [18]. Consistently, flies with ablated insulin-producing cells have increased levels of lipids and starvation resistance [35]. Flies with reduced rpd3 levels have also increased levels of glucose, glycogen, and trehalose at older ages, illustrating increased energy storage [18]. It is also possible that increased resistance to starvation observed in $r p d 3$ flies results from metabolic adaptation to nutri- ent availability. Such changes could be mediated by changes in acetylation of metabolic enzymes, which could affect their enzymatic activity allowing a shift from one metabolic pathway to another [36, 37]. It was shown that histone modifying enzymes link changes in nutrient availability to changes in intermediary metabolism by affecting the activity of the enzymes involved in glycogenesis, glycolysis, gluconeogenesis, and $\beta$-oxidation through acetylation $[36$, 37].

DR has no effect on oxidative stress resistance early in life but decreases resistance to paraquat as flies age [28]. We showed that $r p d 3$ reduction increases resistance to oxidative stress, as measured by increased resistance to $\mathrm{H}_{2} \mathrm{O}_{2}$. This resistance is mediated by reduced IIS, illustrated by increased dFoxo mRNA levels found in rpd3 mutant flies, and lack of increased resistance to $\mathrm{H}_{2} \mathrm{O}_{2}$ and shorter lifespan observed in flies double mutant for $d F$ Foxo and $r p d 3$, compared to single rpd3 mutant flies [18]. This is in agreement with findings that overexpression of nuclear localized dFoxo mediates increased resistance to oxidative stress in flies [38]. Furthermore, flies treated with PBA, a HDAC1 inhibitor, have increased expression of genes that have been implicated in response to oxidative stress such as glutathione S-transferase and SOD [16]. PBA-treated flies have increased climbing activity, increased resistance to starvation and to paraquat, and extended lifespan [16].

Several findings presented here further confirm that rpd3 reduction extends longevity by a mechanism that partially overlaps with DR but has also distinct characteristics. $r p d 3^{\text {def }}$ male flies have an increased peak of spontaneous physical activity. However, $r p d 3$ flies have no changes in fecundity, total spontaneous physical activity, and are more resistance to starvation and oxidative stress compared to controls. Our data illustrates how complex interactions between reduced $r p d 3$ levels and its downstream targets results in various phenotypic changes at the organismal level.

\section{Acknowledgments}

We would like to thank Suzanne Kowalski, Alexander Pokorski, Bhavin Gupta, and Ryan Rogers for their help in the lab. We thank Dr. Stewart Frankel for critical reading of the manuscript. This work was supported by grant from the NIH (AG023088 to B.R.) 
and UCONN Health (to B.R.). B. Rogina is a recipient of a Glenn Award for Research in Biological Mechanisms of Aging.

\section{References}

[1] Weindruch RH, Walford RL. The Retardation of Aging and Disease by Dietary Restriction. Springfield, IL, C.C. Thomas. 1988.

[2] Fontana L, Partridge L. Promoting health and longevity through diet: From model organisms to humans. Cell. 2015;161(1): 106

[3] Fontana L, Coleman RJ, Holloszy JO, Weindruch R. Calories restriction in non-human and human primates. In Handbook of The Biology of Aging. Eds. EJ Masoro and SN Austad. Academic Press. 2010, p. 447.

[4] Seto E, Yoshida M. Erasers of histone acetylations: The histone deacetyase enzymes Cold Spring Harb. Perspect Biol. 2014;6:a08713.

[5] Murphy M, Ahn J, Walker KK, Hoffman WH, Evans RM, Levine AJ, George DL. Transcriptional repression by wild-type p53 utilizes histone deacetylases, mediated by interaction with mSin3a. Gen Devel. 1999;13(19):2490.

[6] Juan LJ, Shia WJ, Chen MH, Yang WM, Seto E, Lin YS, Wu CW. Histone deacetylases specifically down-regulate p53-dependent gene activation. J Biol Chem. 2000;275(27): 20436.

[7] Woods J, Rogina, B. The effects of Rpd3 on fly metabolism, health and longevity. Exp Gerontol. 2016;8(11):3028.

[8] Marks A, Breslow R. Dimethyl sulfoxide to vorinostat: Development of this histone deacetylase inhibitor as an anticancer drug. Nature Biotechnol. 2007;25(1):84.

[9] Ganai SA, Ramadoss M, Mahadevan V. Histone deacetylase (HDAC) inhibitors - emerging roles in neuronal memory, learning, synaptic plasticity and neural regeneration. Curr Neuropharmacol. 2016;14(1):55.

[10] Kim S, Benguria CY, Lai SM, Jazwinski M, Modulation of life-span by histone deacetylase genes in Saccharomyces cerevisiae. Mol Biol Cell. 1999;10:3125.

[11] Rogina B, Helfand SL, Frankel S. Longevity regulation by Drosophila rpd3 deacetylase and caloric restriction. Science. 2002;298(5599):1745.

[12] Pallos J, Bodai L, Lukacsovich T, Purcell JM, Steffan JS, Thompson LM, Marsh JJ. Inhibition of specific HDACs and sirtuins suppresses pathogenesis in a Drosophila model of Huntington's disease. Hum Mol Genet. 2008;17(23): 3767.

[13] Rogina B, Helfand SL. Sir2 mediates longevity in the fly through a pathway related to calorie restriction. Proc Natl Acad Sci USA. 2004;101(45):15998.

[14] Frankel S, Woods J, Ziafazeli T, Rogina B. RPD3 histone deacetylase and nutrition have distinct but interacting effects on Drosophila longevity. Aging. 2015;7(12):1112.

[15] Kopp ZA, Hsieh JL, Li A, Wang W, Bhatt DT, Lee A, Kim SY, Fan D, Shah V, Siddiqui E, Ragam R, Park K, Ardeshna D, Park K, Wu R, Parikh H, Parikh A, Lin YR, and Park Y. Heartspecific Rpd3 downregulation enhances cardiac function and longevity. Aging. 2015;7(9):648.
[16] Kang H-L, Benzer S, Min K-T. Life extension in Drosophila by feeding a drug. Proc Natl Acad Sci USA. 2002;99(2):838

[17] Bauer J, Antosh M, Chang C, Schorl C, Kolli S, Neretti N, Helfand SL. Comparative transcriptional profiling identifies takeout as a gene that regulates life span. Aging. 2010;2(5) 298.

[18] Woods J, Ziafazeli T, Rogina B. Rpd3 interacts with insulin signaling in Drosophila longevity extension. Aging. 2016;8(11):3028.

[19] Gershman B, Puig O, Hang L, Peitzch RM, Tatar M, Garofalo RS. (2007) High-resolution dynamics of the transcriptional response to nutrition in Drosophila: A key role for $\mathrm{dFOXO}$ Physiol Genomics. 2007;29(1):24.

[20] Mottus R, Sobel, RE, Grigliatti TA. Mutational analysis of a histone deacetylase in Drosophila melanogaster: Missense mutations suppress gene silencing associated with position effect variegation. Genetics. 2000;154(2):657.

[21] Woods J, Kowalski S, Rogina B. Determination of the spontaneous locomotor activity in Drosophila melanogaster. J Vis Exp. 2014;86:1-6. doi: 10.3791/51449

[22] Bross TG, Rogina B, Helfand SL. Behavioral, physical, and demographic changes in Drosophila populations through dietary restriction. Aging Cell. 2005;4(6):309.

[23] Rogina B, Wolverton T, Bross TG, Chen K, Müller H-G, Carey JR. Distinct biological epochs in the reproductive life od female Drosophila melanogaster. Mech. Aging Dev. 2007;128(9):477.

[24] Rogers RP, Rogina B. Increased mitochondrial biogenesis preserves intestinal stem cell homeostasis and contributes to longevity in Indy mutant flies. Aging. 2014;6(4):335.

[25] Parashar V, Rogina B. dSir2 mediates the increased spontaneous physical activity in flies on calorie restriction. Aging. 2009:1(6), 529.

[26] Parashar V, Rogina B. dSir2 and fly mobility. Cell Cycle. 2010;9(3):433.

[27] Chen D, Steele AD, Lindquist S, Guarente L. Increase in activity during calorie restriction requires Sirt1. Science. 2005;310(5754):1641

[28] Burger JM, Hwangbo DS, Corby-Harris V, Promislow DE. The functional costs ad benefits of dietary restriction in Drosophila. Aging Cell. 2007;6(1):63.

[29] Rion S, Kawecki TJ. Evolutionary biology of starvation resistance: What we have learned from Drosophila. J Evol Biol. 2007;20(5):1655.

[30] Piper MD, Skorupa D, Partridge L. Diet, metabolism and life span in Drosophila/Exp. Gerontol. 2005;40(11):857.

[31] Wang PY, Neretti N, Whitaker R, Hosier S, Chang C, Lu D, Rogina B, Helfand SL. Long-lived Indy and calorie restriction interact to extend life span. Proc Natl Acad Sci USA. 2009;106(23):9262-9267.

[32] Parashar V, Frankel S, Lurie AG, Rogina B. The effects of age on radiation resistance and oxidative stress in adult Drosophila melanogaster. Rad Res. 2008;169(6):707.

[33] Skorupa DA, Dervisefendic A, Zwiener J, Pletcher SD. Dietary composition specifies consumption, obesity, and lifespan in Drosophila melanogaster. Aging Cell. 2008;7(4): 478.

[34] Djawdan M, Chippindale AK, Rose MR, Bradley TJ. Metabolic reserves ad evolved stress resistance in Drosophila melanogaster. Physiol Zool. 1998;71(5):584. 
[35] Broughton SJ, Piper MD, Ikeya T, Bass TM, Jacobson J, Driege Y, Martinez P, Hafen E, Withers DJ, Leevers SJ, Partridge L. Longer lifespan, altered metabolism, and stress resistance in Drosophila from ablation of cells making insulin-like ligands. Proc Natl Acad Sci USA. 2005;102(8): 3105.

[36] Guan KL, Xiong Y. Regulation of intermediary metabolism by protein acetylation. Trends Bioch Sci. 2011;36(2):108.

[37] Zhao S, Xu W, Jiang W, Yu W, Lin Y, Zhang T, Yao J, Zhou L, Zeng Y, Li H, Li Y, Shi J, An W, Hancock SM, He F, Qin L,
Chin J, Yang P, Chen X, Lei Q, Xiong Y, Guan KL. Regulation of cellular metabolism by protein lysine acetylation. Science. 2010;3275968 1000 .

[38] Jünger MA, Rintelen F, Stocker H, Wasserman JD, Végh M, Radimerski T, Greenberg ME, Hafen E. The Drosophila Forkhead transcriptional factor FOXO mediates the reduction in cell number associated with reduced insulin signaling. J Biol. 2003;2:20. 